\title{
La política educativa para la enseñanza de la historia de Colombia (1948-1990): de los planes de estudio por asignaturas a la integración de las ciencias sociales
}

\section{//Education policies for teaching Colombian \\ history (1948-1990): From curricula for subjects \\ to Social Sciences involvement}

//A política educativa para o ensino da história da Colômbia (1948-1990): dos planos de estudo por disciplinas à integração das ciências sociais de Educación, N. 62.
Primer semestre de 2012,
Bogotá, Colombia.

\begin{abstract}
Doctor en Historia de la Universidad de Huelva, magister en Historia de la Universidad Industrial de Santander, especialista en Filosofía de las Ciencias de la Universidad de Antioquia e historiador de la Universidad Industrial de Santander. Docente de la Universidad Industrial de Santander, vinculado al grupo de investigación Políticas, sociabilidades y representaciones histórico-educativas (PSORHE) en la línea Manuales escolares e historia de la Educación. Correo electrónico: tarazona20@gmail.com

Historiador y estudiante de maestría en Historia de la Universidad Industrial de Santander. Docente-investigador de la Universidad Industrial de Santander, vinculado al grupo Políticas, sociabilidades y representaciones histórico-educativas. Correo electrónico: davidsalon16@ gmail.com
\end{abstract}

\section{Resumen}

Este artículo se ocupa de abordar la política educativa relacionada con la invención de una memoria histórica que el Estado colombiano desarrolló y trató de implementar durante la segunda mitad del siglo XX. El planteamiento central que se sostiene en estas cuartillas es que el Estado central sí se interesó por incidir en la definición de la memoria compartida desde una perspectiva que podría denominarse nacionalista, más allá de los cambios a nivel pedagógico que se acometieron en el periodo de estudio. La nación fue el referente central del proyecto de memoria compartida del Ministerio de Educación, al punto que se mantuvo en el proceso de transición de la enseñanza de las asignaturas separadas del proyecto integrado de mediados de los años ochenta. El propósito era mantener los rasgos propios de un nacionalismo cultural articulados con una formación política y cívica legitimadora del régimen democrático vigente y del sistema capitalista como meta deseable de toda la sociedad. Este artículo se basó fundamentalmente en el estudio de la documentación oficial expedida por el MEN con el fin de analizar los marcos educativos que tuvo la producción de manuales escolares en la segunda mitad del siglo XX.

\section{Palabras Clave}

Política educativa, enseñanza de la historia, memoria nacional, ciencias sociales. 


\section{Abstract}

This paper focuses on addressing educational policies related to invention of a historical memory that Colombian state has developed and tried to implement during the second half of the 20th century. The main approach in these pages is that central state was really interested to influence a definition of shared memory from a deeper national perspective, regardless of educational level changes undertaken in this study period. So nation was the focus of the shared memory project by Ministry of Education, so during transition from teaching separate subjects to integrated project in the mid-eighties was kept. The purpose was to maintain distinctive features of a cultural nationalism linked to a political and citizenship education legitimizing the current democratic regime and a capitalist system as a desirable goal of any society. This paper is based primarily on a study of official documents issued by MEN in order to analyze the educational standards focused on textbook production during the second half of the 20th century.

\section{Keywords}

Educational policies, teaching history, national memory, Social Sciences.

\section{Resumo}

Este artigo se dedica a abordar a política educativa relacionada com a invenção de uma memória histórica que o Estado colombiano desenvolveu e implementou durante a segunda metade do século XX. A ideia central que se sustenta nessas cartilhas é que o Estado central se interessou por influir na definição de memória compartilhada a partir de uma perspectiva que se poderia denominar de nacionalista, além das mudanças em nível pedagógico realizadas no período em estudo. A nação foi o tema central do projeto de memória compartilhada do Ministério da Educação Nacional (MEN), a tal ponto que se manteve no processo de transição do ensino das disciplinas separadas do projeto integrado de meados dos anos oitenta. O objetivo era manter as características próprias de um nacionalismo cultural articuladas com uma formação política e cívica legitimadora do regime democrático vigente e do sistema capitalista como meta desejável de toda a sociedade. Este artigo se baseou fundamentalmente no estudo da documentação oficial expedida pelo MEN, com o fim de analisar os marcos educativos que a produção de livros didáticos teve na segunda metade do século XX.

\section{Palavras chave}

Política educativa, ensino da história, memória nacional, ciências sociais. 


\section{Presentación}

La relevancia de la memoria para la vida social parece estar fuera de duda, no solo por la vigencia que tiene el término en las últimas décadas sino por la amplitud de campos en los que es utilizada. Especialmente, la situación del país respecto al conflicto armado y las sucesivas crisis humanitarias han posicionado el asunto de la memoria en la esfera pública, ligándolo a la recuperación de la memoria colectiva de las víctimas de violación de los derechos humanos (CNRR, 2008, 2010; Minga, 2008). Sin embargo, son muy pocos los trabajos en el país que se han interesado por pensar las maneras como el poder político participa en la configuración de la memoria compartida ya sea a nivel nacional o regional (Rincón, 2010). Conmemoraciones, intervención en los espacios públicos con la creación de "lugares de la memoria" y fiestas patrias son algunos de los mecanismos en los que el poder ha intentado moldear los recuerdos de la sociedad colombiana (Acevedo, 2011). En otras ocasiones y de acuerdo a las condiciones del conflicto se han impuesto los pactos de silencio, tal y como lo sugiere Gonzalo Sánchez al hablar del final de La Violencia. (Sánchez, 2006)

Ahora bien, la enseñanza de la historia escolar, y con ella la creación de los programas y planes de estudios o la elaboración de los manuales escolares no han sido inter- pretados como parte de la creación de la memoria oficial. La creación de la identidad nacional o los sesgos segregacionistas a poblaciones específicas como las mujeres o las etnias son las principales preocupaciones de los trabajos emprendidos con base en los manuales escolares (Herrera y otros, 2003). En España y Argentina, los estudios sobre el papel del Estado en la definición de la memoria social cobran mayor interés, tal y como lo muestran los estudios sobre la política educativa durante el Franquismo o las dictaduras militares e incluso en los procesos de transición a la democracia (Pérez Garzón, 2000; Romero, 2004). A pesar de la relevancia y vigencia de este interés temático, pocos se dan a la tarea de decir explícitamente qué entienden por memoria, razón por la cual intentaremos compartir con el lector cómo entendemos esta noción para efectos del trabajo.

Aunque la bibliografía al respecto es inmensa, consideramos a manera de síntesis que la memoria tiene que ver con la definición social y política de aquello que los individuos recuerdan y olvidan (Candau, 2002). En otras palabras, la memoria es una forma de acercarse al "pasado" o "lo que ha sido", que no solo se remite a la experiencia propia que se evoca, la acción que Ricoeur llama rememoración, sino a los conocimientos y valores de unas generaciones que nos precedieron y que generalmente son aprendidas (Ricoeur, 2003). Si bien memoria e historia son diferen- 
tes, no se abstraen de la disputa política y de los intereses que se dan socialmente por su construcción, definición y preservación. En este caso, consideramos que se puede hablar de una "memoria histórica", entendiendo por esta la creación de un conjunto de recuerdos y olvidos por parte del conocimiento histórico, específicamente respecto a los acontecimientos y procesos del tiempo largo que también inciden en las relaciones del presente y la construcción del futuro (Halbwachs, 2004).

Hablar de la memoria no solo como experiencia personal, que desde los trabajos de Maurice Halbwachs sabemos se da en unos marcos sociales, también obliga a pensar en la relación con el poder. De este modo, autores como Anderson nos recuerdan cómo la creación de una nación no sólo implica la consolidación de un Estado moderno sino la invención de una comunidad imaginada (Anderson, 1993). En tal sentido, en cuanto es el Estado el actor principal que mediante sus políticas educativas o culturales pretende definir aquello que se ha de recordar y por ende olvidar en un momento histórico dado, podremos hablar de memorias oficiales, e incluso cuando el grado de aceptación y asimilación es alto entre la población podríamos hablar de memorias hegemónicas o dominantes (Gnecco y Zambrano, 2000). Aunque la relación del poder con la creación de memoria es más compleja y matizada (Sánchez, 2000), en este trabajo nos enfocaremos en el estudio de los planes de estudio para la enseñanza de la historia durante la segunda mitad del siglo XX en el país, como parte del proceso de la memoria oficial gestionada por el Estado.

El planteamiento central que se sostiene en estas cuartillas consiste en que el Estado central sí se interesó por incidir en la definición de la memoria histórica del país desde una perspectiva que podría denominarse nacionalista, más allá de los cambios a nivel pedagógico que se acometieron en el periodo de estudio. La nación fue el referente central del proyecto de memoria compartida del Ministerio de Educación, al punto que se mantuvo en el proceso de transición de la enseñanza de las asignaturas separadas al proyecto integrado de mediados de los años ochenta. El propósito era mantener los rasgos propios de un nacionalismo cultural articulados con una formación política y cívica legitimadora del régimen democrático vigente y del sistema capitalista como meta deseable de toda la sociedad. Este artículo se basó fundamentalmente en el estudio de la documen- 
tación oficial expedida por el MEN con el fin de estudiar los marcos educativos que tuvo la producción de manuales escolares en la segunda mitad del siglo XX.

\section{De la historia patria remozada a la historia nacionalista velada}

Iniciada con la creación de la Academia de Historia a principios del siglo XX en plena guerra civil de los Mil Días, la historia patria experimentó una reedición luego de los aciagos sucesos del 9 de abril de 1948 (Velandia, 2001; Betancourt, 2007; Tovar, 1982, 1984). Convencidos ciertos sectores de la élite política e intelectual de que los desmanes fueron producto del olvido de las obras y espíritus de los grandes hombres de la Patria, el gobierno nacional expidió el Decreto 2388 por el que se intensificaba la enseñanza de la historia patria (Presidencia de la República, 15 de julio de 1948). Con el objetivo de la cohesión nacional y la dignificación de la ciudadanía colombiana, se consideró que la educación cumpliera el papel de formadora de hábitos democráticos, decoro personal y orgullo nacional a través de la historia. Una de las medidas tomadas para concretar estos propósitos fue la declaración de obligatoriedad del estudio de la Historia de Colombia en todos los grados, estableciendo como mínimo dos grados tanto en primaria como en secundaria.
La selección de profesores con mentalidad suprapartidista o la elaboración de todos los programas de enseñanza fueron algunas de las medidas que contemplaba esta nueva normatividad. Para ello contó con la asesoría de la Academia Colombiana de Historia $(\mathrm{ACH})$, a quien se le otorgaron funciones como la autorización de los textos escolares a emplear en las aulas, la preparación de material literario y audiovisual, la realización de la Cátedra Superior de Historia de Colombia y la participación en premiaciones oficiales creadas por el MEN. La Cátedra de Historia fue considerada por la $\mathrm{ACH}$ como la "misión" más delicada de todas las impuestas por el MEN, a la que se dirigirían los esfuerzos para contribuir a mejorar la enseñanza de la historia. Considerada como "superior", la cátedra debía tener el nivel de conocimiento mínimo del programa dirigido al grado $6^{\circ}$ del bachillerato (García Samudio, 1948)' 1 .

El proyecto de extender la historia patria a todo el sistema educativo no solo se refería a la priorización como asignatura, sino que se acom-

\footnotetext{
Las temáticas contempladas en este espacio formativo para docentes consistieron en un recorrido por la historia política de la nación así: causas internas y externas de la Independencia y Guerra de Independencia; Gran Colombia; Nueva Granada y Confederación Granadina; Estados Unidos de Colombia y República de Colombia Para el desarrollo de la cátedra, se consideró que cada periodo fuese tratado en diez clases a cargo de cinco o seis académicos en un lapso de ocho meses, mientras que la administración de presupuesto y coordinación correría por cuenta del secretario y el bibliotecario de la $\mathrm{ACH}$.
}

,


pañó de la difusión del patriotismo a partir de varias de estrategias. Entre estas se pueden mencionar los premios en efectivo para los profesores acompañados de diplomas honoríficos, retratos de Santander y Bolívar en cada salón de clase o la consagración de los colegios a un "patrono cívico". El apuntalamiento de una educación cívica centrada en la obediencia a las autoridades fue el objetivo central de la normatividad educativa, redefinida en las enseñanzas del pasado. (Presidencia de la República, 15 de julio de 1948).

Un par de meses después de la expedición del decreto se lanzó un concurso dirigido a los planteles femeninos para que las señoritas escribieran textos de historia patria (MEN, 6 y 21 septiembre de 1948). El proyecto llevó a la creación de la Medalla Cívica Camilo Torres como homenaje de la república a los educadores consagrados al enaltecimiento de la patria. El nombre de la medalla se debía a los valores que representó este prócer para la historia, símbolo de la libertad, la democracia y la justicia que los maestros estaban llamados a consolidar con su quehacer pedagógico. Los valores de los docentes que se premiarían con este reconocimiento eran la conducta impoluta, la perseverancia, el compañerismo y el espíritu apostólico que caracterizaba la profesión. Quienes obtuvieran la medalla serían acreedores a una serie de beneficios laborales como ascensos, predilección para acceder a becas, entre otros (Presidencia de la República, 6 de octubre de 1948) ${ }^{2}$.

Con este tipo de iniciativas que se complementaron con eventos como el cambio de nombre de las entidades educativas para "bautizarlas" con el de prohombres de la patria, el Estado central pretendió crear la concordia nacional fracturada, según dicho propósito luego de los sucesos aciagos de 1948. (MEN, 9 de octubre de 1948). La vuelta a la historia patria y su alto componente de formación cívica puede ser entendida como un intento desesperado por canalizar, a través del sistema educativo, unos valores que disminuyeran la confrontación bipartidista y persuadieran al "pueblo" de la impertinencia de levantarse para desconocer las jerarquías seculares que tenían que existir. Todos los colombianos, sin distingo de color ni clase, debían arroparse bajo la idea

2 La historia patria también debía contemplarse en las izadas de bandera y los discursos de los directores de los colegios con alto contenido cívico. El empleo de medios como la Radiodifusora Nacional para la promoción de la historia nacional fue contemplado para los mismos fines. 
de patria, siendo la historia escolar un escenario privilegiado para insuflar este sentimiento.

Como se sabe, la segunda mitad del siglo XX presenció la explosión de procesos y tendencias modernizadoras en varios órdenes en el país. La revitalización de un capitalismo más industrial propició el aumento de la matrícula en todos los niveles del sistema educativo, además de procesos urbanizadores no planificados a la par del mejoramiento de indicadores como los de mortalidad, natalidad, nutrición y analfabetismo (Palacios, 2003, p. 289-330). Esta salud favorable de la economía colombiana y un mejoramiento de la calidad de vida de la población en las ciudades, especialmente de la zona cafetera, no obstante contrastaba en las zonas rurales donde la reforma agraria del año 36 había acentuado los conflictos entre propietarios y campesinos sin tierra.

La modernización como fenómeno urbano no llegaba a las zonas rurales, donde aún en 1945 se concentraban las dos terceras partes de los colombianos. Un $60 \%$ no sabía leer y las brechas de calidad de vida entre las clases pudientes y las rezagadas eran notorias. Las tierras cafeteras de Antioquia, Tolima y Valle eran un atractivo de oportunidades para miles de campesinos que abandonaban el oriente y el sur de un país con unos 10.5 millones de habitantes. Las tasas de mortalidad infantil decrecían al mismo tiempo que la de analfabetismo, y el promedio de vida se elevó de 36.1 años a 48.9 años. En general, en la década del cuarenta había signos de una mejora en las condiciones de vida de los colombianos en las ciudades, relacionadas con acceso a médicos, hospitales, profesores y escuelas. Unas mejorías que contrastaban con las zonas rurales del país, sumidas en la escasez, la explotación de los propietarios de las tierras y la violencia bipartidista entre liberales y conservadores, azuzada esta última desde Bogotá y las capitales departamentales por caciques, caudillos, gamonales y políticos de oficio. En estas condiciones tan desiguales entre el campo y las ciudades colombianas nacía la clase media en las zonas más urbanizadas y con ellas nuevas formas de consumo.

En este marco de contrastes, el proyecto educativo general inició un largo proceso -todavía no concluido- de adecuación educativa para las nuevas generaciones en el paradigma del desarrollo y el fortalecimiento del sistema democrático (Martínez Boom, 2004; MEN, 22 de enero de 1976 y 17 de julio de 1978). Ahora, surge la pregunta: ¿Cuál fue el lugar atribuido al conocimiento social en este proceso reformador?

Con el Decreto 045 de 1962, el MEN expidió en 1963 los Programas analíticos de estudios sociales y Filosofía para el primer y segundo ciclo de educación media. El proyecto de enseñanza de los estudios sociales representó un momento de tránsito 
entre los postulados centrados en promover el sentimiento patriótico y aquella tendencia que quería darle al conocimiento social mayor impacto en la construcción de la democracia y el desarrollo. A partir de este momento se empezará a dar un lento distanciamiento de los postulados patrióticos para engranar el conocimiento histórico y geográfico en los paradigmas citados.

En plena Guerra Fría, el Estado colombiano pretendió brindar a los alumnos una formación espiritual, moral y cívica que permitiera una convivencia armónica no solo al interior de la nación sino con el mundo. Sin embargo, la invención de continuidades con el pasado más remoto no se hizo esperar, tal y como se concebía en la asignatura de "Prehistoria General, Americana y de Colombia". Para ello, se esperaba: "Formar en el alumno la conciencia de que la sangre y los sentimientos de los indios están vivos en nuestro pueblo y de cómo es indispensable, para el progreso de Colombia, comprender todas las tendencias y pasados que se fundan en nosotros" (MEN, 1963, p. 14-15).

Junto a la promoción del orgullo por el pasado prehispánico, se esperaba socializar a los estudiantes en la idea de una comunidad americana. Para tal efecto, se centraban los programas en la "epopeya de la emancipación", entendida como una gloria común de los pueblos del Nuevo Mundo. De allí que los contenidos históricos para el tercer año buscaran inocular una fidelidad al mandato de los fundadores de la nacionalidad cultivando el sentimiento de solidaridad continental (MEN, 1963, p. 38). El acento puesto en la idea de una América como unidad continental fue una novedad respecto a la historia patria de la primera mitad del siglo. Este enfoque se mantendrá décadas más adelante.

Pese al cambio de intenciones, el patriotismo se mantuvo tras el velo de la historia nacional, tal y como se puede ver en los objetivos trazados:

Cultivar el sentimiento de la nacionalidad, apoyándolo en la triple base de amor, comprensión y servicio a la patria. Hacer percibir las esencias del alma colombiana, su génesis y sus manifestaciones características. Robustecer el amor a la patria en el conocimiento de sus glorias y por la exaltación de sus héroes consagrados. Destacar el sentido heroico [sic] de las grandes luchas por la libertad y, a propósito de la narración de éstas, despertar la admiración hacia las figuras de los libertadores, por su patriotismo, espíritu de sacrificio en bien del 
pueblo, su perseverancia en superar los obstáculos que se les presentaron y ofrecer como ejemplo las virtudes que adornaron su carácter. Destacar la importancia de los sacrificios que hizo el pueblo para lograr su independencia. Hacer ver que la emancipación fue el resultado de factores humanos, tales como mestizaje, lengua, religión, gobierno, leyes $y$ costumbres, capaces de producir una revolución política. Estudiar la obra y la doctrina de Bolívar, presentar a éste como el Padre de la Patria y el Genio de América, en quien converge toda una época, la encarna, orienta y define. Presentar la figura de Santander como el organizador de la República y como defensor de la justicia y el respeto a la Ley. Presentar las guerras civiles del siglo pasado como resultado de los esfuerzos por restablecer la justicia; hacer notar cómo ellas retardaron el progreso, cómo la paz beneficia a todos y es indispensable para el adelanto material y cultural del país, por lo cual todos los colombianos estamos en el deber de ayudar a conservarla [...] (MEN, 1963, p. 55).

La memoria a configurar se inscribía en la grandes gestas y en la "tradición civilista" de las "instituciones de la República", a las que había que respetar y mantener, no solo por su significado histórico sino porque gracias a ellas, todo colombiano encontraba oportunidades para su vida. Con la Geografía sucedió algo similar, pues se esperaba que con esta asignatura el alumno pudiera alcanzar un conocimiento racional del mundo y con ella una comprensión del medio ambiente y de las problemáticas socioeconómicas de la vida contemporánea. El conocimiento geográfico estaba Ilamado a crear el sentimiento de una nación privilegiada, no ya por las acciones de sus hombres sino por los dones de la naturaleza. En este punto se percibía el interés por potenciar una educación más práctica e industriosa ligada a la promoción de la identidad nacional: Destacar la ventajosa
posición de Colombia
en el mundo en relación
con las comunicaciones,
transporte, comercio y
relaciones internaciona-
les. Hacer hincapié en
la necesidad que tiene
el país de capacitación
para aprovechar cada
vez mejor esa privilegia-
da situación [...] Hacerle 
comprender que, no obstante la multiplicidad de paisajes, debe afianzarse la unidad nacional. [...] Mostrar a los alumnos el proceso de la formación del pueblo colombiano en virtud del mestizaje realizado desde la Conquista, que ha librado al país de los problemas raciales y ha afianzado la UNIDAD NACIONAL. Insistir en cómo el pueblo colombiano, por poseer raíces étnicas de las diversas partes del mundo, está en condiciones de interesarse por la problemática universal. Dar a conocer las enfermedades que afectan nuestro pueblo y disminuyen su vitalidad; enseñarles cómo es posible contrarrestar dichos flagelos mediante las campañas sanitarias y nutricionales e inculcarles hábitos tendientes a la conservación de la salud. Destacar el papel que la Religión, la lengua y el folclore han desempeñado en nuestra cultura a través de los tiempos. [...] Dar a conocer la potencialidad y la realidad económica de Colombia para estimular el patriotismo y formar la conciencia de una explotación racional de los recursos naturales. Establecer las relaciones existentes entre nuestros recursos naturales y forma de aprovechamiento, necesidades y distribución de riquezas. Hacer ver que el bienestar económico del individuo y de la nación es resultante de la capacidad de trabajo del hombre, de la práctica de un ahorro racional y de la acertada administración de la riqueza pública (MEN, 1963, p. 85-89, 91).

Los contenidos de cada una de estas materias no diferían mucho de los tradicionales relacionados con las gestas de la Independencia y la historia político-militar o los rudimentos en Geografía física -especialmente la del territorio nacional- y en menor medida cuestiones "humanas" y culturales. En cuanto a la educación cívica, los estudiantes debían aprender las normas básicas de la convivencia para fortalecer la unidad familiar que redundaría en el fortalecimiento de la patria. De los símbolos nacionales se pretendía arraigar nociones de orden asociadas a la consolidación de la república, con énfasis en principios constitucionales, el Estado como "forma de organización perfecta" y la utilidad social de los futuros ciudadanos. El aprendizaje de estos contenidos tendría que derivar en el respeto a la autoridad y la obediencia a las normas establecidas (MEN, 1963, p. 115-118). 
Para cerrar este apartado, es necesario señalar cómo los programas del año 63 introdujeron formas activas de enseñanza, retomadas en los años ochenta bajo el paradigma de la escuela activa. Al proponer estrategias que no generaran una adquisición mecánica de los conocimientos, el MEN cifraba sus intenciones de modelar ciudadanos democráticos en los mismos métodos:

$$
\begin{aligned}
& \text { Así, la formación del es- } \\
& \text { píritu democrático im- } \\
& \text { plica en los alumnos el } \\
& \text { desarrollo del espíritu de } \\
& \text { comprensión para actuar } \\
& \text { en equipo, espíritu que } \\
& \text { es la base del progreso } \\
& \text { democrático; por consi- } \\
& \text { guiente, el profesor debe- } \\
& \text { rá emplear métodos que } \\
& \text { exijan a los alumnos el } \\
& \text { trabajo en grupos, don- } \\
& \text { de se respeten los dere- } \\
& \text { chos y opiniones de cada } \\
& \text { cual, sienta el individuo } \\
& \text { su responsabilidad fren- } \\
& \text { te al grupo y donde se } \\
& \text { ofrezcan oportunidades } \\
& \text { para el desarrollo de una } \\
& \text { conciencia social (MEN, } \\
& 1963, \text { p. } 7 \text { ). }
\end{aligned}
$$

La propuesta se orientaba al empleo de los llamados "métodos activos", cuyo origen no era otro que los postulados de la Escuela Nueva. (Herrera, 1999). Este modelo centrado en el niño y no en la tarea directiva e instructora del maestro, y promotor de una educación para el cambio social valorando la autoafirmación y la actividad espontánea de los niños, tuvo una renovada incorporación en América Latina y el Caribe luego de la Segunda Guerra Mundial y llegó a alterar, en parte, las propuestas espiritualistas autoritarias, especialmente los enfoques humanistas de las primeras experiencias dictatoriales del Cono Sur.

En el caso de los estudios sociales para Colombia, se planteó la necesidad de articular la Historia y la Geografía junto a la promoción de habilidades como la discusión y el debate sobre temas sociales, suprimiendo estrategias como el dictado o la cátedra magistral. En general, el proceso educativo debía adaptarse a la capacidad mental y a las necesidades del alumno. Propuestas "novedosas" como la observación del entorno inmediato, el acercamiento a los documentos históricos, la comparación entre el pasado y el presente o la utilización de material audiovisual se entrecruzaban con otras "tradicionales" como las "fechas-jalones" o el uso sistemático de las biografías. La enseñanza de la Geografía se basaba en los mismos principios, aunque pretendió introducir otros recursos relacionados con la inventiva, el juego y la creatividad, como los rompecabezas y los "viajes imaginarios" (MEN, 1963, p. 7-12, 19-20, 65-73, 77-80, 86-88, 90 y 109-112).

La valoración del proyecto educativo en ciencias sociales, entre finales de la década del cuarenta y el inicio de los sesenta, se relaciona 
con la continuidad en la orientación de los contenidos patrióticos, que tenían a la nación como el eje del relato histórico, de la representación geográfica y de la formación cívica. Para el caso de la Historia de Colombia la concepción no varió, toda vez que se centró en las gestas de los grandes hombres que con su vida dieron origen a la república, caracterizada como esencialmente democrática. La Geografía, por su parte, se encargaba de unir profundamente a los alumnos al territorio nacional con base en el conocimiento de los rasgos físicos y naturales, las riquezas y potencialidades del suelo patrio manifestadas en los recursos naturales y su población. La educación cívica complementaba la formación de la identidad del alumno con la nación, determinando los comportamientos privados de los alumnos y señalando el camino de la ciudadanía por intermedio de la insistencia en el respeto y obediencia a las autoridades y la ley.

Más allá de la introducción de métodos activos que serían retomados décadas más adelante tanto en los marcos curriculares como en los manuales escolares, el conocimiento social impartido en la escuela colombiana no renunció a la formación de patriotas. A partir de la década del setenta, con el surgimiento de la historia universitaria y sus aportes a una historia más socioeconómica, emergerían nuevos intentos de reforma. Pese a las buenas intenciones, el Estado colombiano mantendría el acento en la nación como eje de la creación de la memoria social aunque con variaciones respecto a la internacionalización de la nación.

\section{La integración de las ciencias sociales: una apuesta inconclusa o el fantasma de la historia patria en la internacionalización}

A mediados de los años setenta se inició un proceso de reforma de la educación colombiana, tanto a nivel institucional como curricular (Herrera y Martínez, 2002). El conocimiento social no fue ajeno a esta ola de cambios, especialmente al involucrar algunos planteamientos de la historia universitaria a su proyecto educativo, tal y como lo muestra el documento de trabajo dirigido al MEN por Jorge Orlando Melo y Jaime Jaramillo Uribe. El texto de Melo y Jaramillo adquiere relevancia porque contiene una serie de recomendaciones que incidirían en la reforma curricular derivada del Decreto 1002 de 1984. Si bien se pretendió dar un 
viraje en la relación entre sistema educativo y productores del conocimiento histórico y social, los marcos curriculares permanecieron invariables en los enfoques de la historia política, incorporando parcialmente las propuestas de los historiadores profesionales.

Las recomendaciones hechas por estos historiadores al MEN para mejorar la enseñanza de la historia se centraron en cuatro aspectos: objetivos, sugerencias metodológicas, contenidos y bibliografía. En materia de objetivos, el documento sugería una historia nacional más integrada al mundo, con el fin de crear una "conciencia nacional firme y positiva". Esta dimensión nacional de la historia armonizaría con valores universales como la solidaridad humana, enfoque necesario en un mundo cada vez más interdependiente que buscaba el bienestar y el progreso del hombre. La Historia tendría que ocuparse de la formación en valores "morales, vitales y científicos" como la tolerancia, el amor a la verdad, el sentido de la realidad y el rigor para emitir juicios sobre personas, colectivos y circunstancias. El nuevo objetivo de la Historia era la preparación de las nuevas generaciones para actuar en una democracia participante.

En la propuesta metodológica los autores compartían la tesis de una educación centrada en el alumno y sus capacidades con el fin de trascender la acumulación insulsa de información. Anticipan- do lo que se daría una década más tarde, insistían que no era posible enseñar Historia alejada de las otras ciencias sociales, especialmente la relación con la Geografía, con el fin de comprender las sociedades en el tiempo y en el espacio. Puntualmente, propusieron algunas habilidades que debía tener el profesor de Historia, entre las que se destacaban: tener un dominio en el acervo importante de anécdotas históricas sin abandonar la mirada de procesos, poseer dotes de dibujante y emplear la imagen como medio para la enseñanza. Además, presentaban el imperativo de ser una persona de espíritu libre, abierto y antidogmático, que impediría que la Historia se prestara para ser escenario de proselitismo político partidista.

En cuanto a los contenidos, la propuesta sí contempló nuevos temas relacionados con los procesos socioeconómicos de la nación y, en menor medida, incorporó temas de la cultura. La historia política no fue desterrada aunque sí se matizó al contextualizarla en el marco de la historia continental y mundial, especialmente cuando se referían a la Independencia. El siglo XIX se sintetizó en la sucesión de guerras civiles y cartas constitucionales con el fin de relievar las dificultades para consolidar una república liberal y democrática. Otros temas considerados relevantes para la enseñanza de la historia fueron el origen de los partidos políticos, los esfuerzos por la industrialización y los transportes, 
temas que de una u otra manera pretendían desplazar la mirada del amor a la patria hacia el desarrollo económico como eje de la historia nacional.

Finalmente, el documento de trabajo presentado al MEN a inicios de los setenta contempló una bibliografía que facilitaría la elaboración de los nuevos marcos curriculares. Esta tendía a priorizar la producción de la "Nueva Historia" sin abandonar las compilaciones documentales de la historia tradicional académica (Melo y Jaramillo, 1971). Este texto representó una propuesta de tránsito moderado en la enseñanza de la historia en el país. Si bien acudió a dos connotadas figuras de la historia universitaria, los objetivos trazados no diferían sustancialmente de los preconizados en tiempos de la historia patria. La nación y especialmente su acontecer político seguían manteniendo un lugar destacado en los contenidos prescritos. La idea de la integración entre Historia y la Geografía mantuvo una postura favorable respecto a una metodología más activa, direccionamiento que se sancionaría oficialmente a mediados de la siguiente década.

\section{Las ciencias sociales integradas: la apuesta por la diversidad y la paz democrática}

Enrumbado en la política de mejoramiento de la calidad educativa, el MEN elaboró un discurso que fundamentara filosófica, pedagógica y políticamente la transformación educativa que el país necesitaba -según el gobierno- para enfrentarse a los nuevos tiempos. Brevemente, la integración se basó en los principios de la descentralización y la búsqueda del desarrollo socioeconómico regional. La vida en democracia y el desarrollo material eran los hilos conductores de todo el sistema educativo, que no abandonaba la misión de fortalecer la nacionalidad, al proponer:

Identificar los valores de la sociedad colombiana. Tomar conciencia de la necesidad de conservar y de enriquecer los auténticos valores de nuestra nacionalidad. Respetar los valores propios y de los de las diferentes culturas como un medio para mejorar las relaciones humanas [...] Desarrollar actitudes que propicien en el individuo un auténtico espíritu nacionalista. (Burgos, 1984, p. 21-22).

Para los años ochenta, el MEN consideraba que ad portas de una cultura mundial, el contexto en que se desenvolvía el proceso educativo estaba dominado por la ciencia y la técni- 
ca, marco de ineludible interés para pensar el currículo en el sentido de adaptar los avances de la ciencia a la realidad nacional. Precisamente, la apuesta del Estado era fortalecer el vínculo de la educación con el sistema productivo para que así se pudieran superar las penurias materiales de la mayoría de colombianos. Los fundamentos psicológicos del proyecto de reforma estaban relacionados con la psicología del desarrollo humano. Concretamente, se pretendía fortalecer en el alumno la psicomotricidad, el desarrollo intelectual y la dimensión socioafectiva $^{3}$. La formación integral del alumno no tenía otro sentido que la preparación óptima para el mundo de la producción, en un tiempo donde se tendía a la "diversificación de la producción y especialización del conocimiento":

En ese sentido la educación de hoy busca preparar al educando para que se integre a la producción material, intelectual y cultural de la sociedad en la cual se desarrolla.

3 A nivel pedagógico la propuesta de integración del MEN retomó postulados de autores como Piaget, Skinner, Bandra y Gagne. Estas teorías del aprendizaje humano se articularon a la propuesta de la pedagogía activa, que desde inicios del siglo XX se pretendió implementar en el país. El énfasis estaba puesto en el niño como sujeto protagónico del proceso de enseñanzaaprendizaje, especialmente en las actividades que este pudiera desarrollar para convertirse en un sujeto autónomo en conexión con la realidad circundante. Los manuales escolares harían eco fuertemente de esta propuesta al dedicar gran parte de sus páginas a actividades evaluativas.
Se educa para producir como sociedad, como grupo, como gremio, como individuo integrado a un sistema social, incluso, aún, en el caso en que no se esté de acuerdo con el sistema, ya que las críticas y oposiciones bien orientadas llevan a una superación de las condiciones que limitan la producción material, intelectual y cultural (Burgos, 1984, p. 50).

En el mismo orden de justificación, los tiempos históricos marcaban la pauta para que el acercamiento al conocimiento dejara de ser fragmentario y disperso. La integración fue la versión educativa del desafío interdisciplinario en boga, siendo la pedagogía activa la manera en que se pretendía llevar a cabo:

La interdisciplinariedad y la integración invaden cada día más los escenarios educativos, a manera de recursos pedagógicos indispensables para los nuevos procesos de enseñanza-aprendizaje. De este modo, la enseñanza y el aprendizaje atomizados y aislados de cada asignatura han ido cediendo terreno a la enseñanza y el aprendizaje integrado, correlacionado, globalizado 
o unificado de varias disciplinas en forma de áreas, o de bloques, donde se busca analizar la interrelación dinámica de varias asignaturas en el manejo interdisciplinario de temas, proyectos, problemas y experiencias concretas de aprendizaje. Ante el peligro que se corre con la división y la especialización de la producción material e intelectual, ante el peligro de que la percepción centrada en un solo árbol no deje ver el bosque, la sociedad moderna propone la enseñanza integrada e interdisciplinaria, donde una misma situación sea analizada y manejada desde varias perspectivas conceptuales. La enseñanza integrada e interdisciplinaria surge así como un reflejo de los modernos métodos de investigación y de los modernos métodos de producción. En efecto, en el mundo actual son cada vez más ilustrativos los casos de investigaciones y de producciones donde la interdisciplinariedad y la integración de conocimientos provenientes de diversas disciplinas sobresalen como ejemplos dignos de imitación, tal es el caso de los proyectos ecológicos, de los proyectos de viajes interplanetarios, de los estudios sobre genética, y de muchos otros ejemplos con los cuales se han de beneficiar los educandos de las actuales y de las futuras generaciones (Burgos, 1984, p. 51).

Sin definir explícitamente aquello que debía entenderse por integración del conocimiento, el MEN, con la asesoría de la Unesco, proponía tres niveles de integración: en el interior de las áreas de conocimiento, entre diferentes asignaturas para llegar finalmente a la disolución de las barreras disciplinares. Como justificación del enfoque integrador se arguyeron razones psicológicas y sociales para optar por una mirada globalizadora de la educación. Tanto el Ilamado "sincretismo infantil" como la complejidad de la realidad social se convirtieron en las razones fundamentales que otorgaban pertinencia a este proyecto (Dirección general de capacitación y perfeccionamiento docente, 1984 , p. 4).

El proyecto integrador que daría forma al currículo debía concretarse en la producción y puesta en práctica de las Ilamadas Unidades Integradas, entendiendo por estas el conjunto de actividades reales organizadas pedagógicamente alrededor de un eje, con el fin de facilitar el aprendizaje integrado y contribuir a la formación unificada. Los criterios pedagógicos a tener en 
cuenta consistían en el reconocimiento de la lógica interna de las ciencias y disciplinas a enseñar y los valores, actitudes y habilidades que se pudieran promover desde ellas. La localidad y el plantel educativo eran pensados como los escenarios fundamentales para llevar a la práctica el proyecto integrador, lo cual no quería decir que el Estado central desapareciera de la planeación educativa, puesto que los fines y enfoques sobre la educación eran del resorte del MEN4.

Elaborados inicialmente para la educación primaria, los marcos generales y los programas curriculares fueron elaborados por reconocidos profesionales de las ciencias sociales y por experimentados licencia$\operatorname{dos}^{5}$. Para el caso de las ciencias sociales (CC.SS), el objetivo central fue el de acompasar los últimos logros en el saber especializado con la comprensión de la realidad nacional por parte de los educandos. Esta pretensión se fundaba en el conocimiento de la vida cotidiana

4 Esto se puede confirmar con la expedición del Decreto 1002 de 1984, a través del que se crearon oficialmente las ciencias integradas, tanto naturales como sociales.

5 Por parte del MEN como programadoras estaban Mercy Abreu de Armengol, Nedgidia Fernández Lara, Rosario Jaramillo de Gamboa, Ana Victoria Navarro. Como docentes colaboradores estaban: por el Quindío, Mariela Arias; por Antioquia, Flor Alzate; por Bogotá, Hugo Prieto y por el Valle del Cauca, José Ignacio Zamudio. Dentro de los asesores se encontraban académicos reconocidos como Gonzalo Hernández de Alba y Camilo Domínguez (UN), José Antonio Ocampo (Fedesarrollo), Marco Palacios (Banco Popular), Rodrigo Parra Sandoval (UPN), Carlos Ernesto Pinzón y Rosa Suárez de Pinzón (IA) y Dora Rothlisberger (Uniandes). como base para el aprendizaje de las habilidades científicas. Si la formación para la democracia era uno de los propósitos fundamentales del proyecto educativo, las ciencias sociales eran el campo de saber específico para lograr tal meta.

La creación de actitudes democráticas e industriosas partía de la nación como un referente ineludible. Sin embargo, se pretendió introducir la idea de diversidad cultural, tanto a nivel externo como hacia el interior:

Comprender la tradición cultural pasada y presente de las diversas regiones del país y de otras partes del mundo para desarrollar una conciencia sana de nacionalidad al tiempo que un aprecio por otras nacionalidades. Entender la identidad colombiana como una unidad que congrega la diversidad de formas de ser que coexisten en el país, que tienen su origen tanto en las diferencias regionales como en la presencia simultánea de lo tradicional y lo moderno [...]Se busca, por tanto, que el alumno tome conciencia de las fuerzas sociales, culturales, regionales, que entran $y$ han entrado en juego para que él, su familia, su región, su nación lleguen 
a ser lo que son, es decir, que el alumno tome conciencia del proceso histórico-cultural en el que se encuentra inmerso, con el fin de que descubra cómo se han creado las relaciones que guarda consigo mismo, con su entorno inmediato, con su región, con la nación y con el mundo. Así, el alumno podrá explicarse con claridad, sus semejanzas y sus diferencias con relación a las de su comunidad y a las de las diversas culturas que coexisten históricamente con él. Estas diversidades, la diversidad de formas y la diversidad de lo económico no se presentan aisladas [...] Esta coexistencia (de formas diferentes de ver la vida, de valorar los hechos) que se da dentro del país, al interior de las regiones, localidades y aun dentro de las familias, es una de las características centrales que definen la identidad colombiana en la época en que vivimos. Por eso, la identidad nacida de la coexistencia de la diversidad, la capacidad de convivencia con personas o grupos que tienen una visión diferente del mundo es un elemento clave para entender la nacionalidad y una herramienta conceptual y valoratoria de gran importancia en la búsqueda de la paz (Abreu, 1988, p. 12, 16).

El conflicto armado que ha marcado la historia reciente el país, agudizado en aquel entonces por la emergencia de la violencia paramilitar y el desenfreno del narcoterrorismo, se convirtió en el telón de fondo de la nueva propuesta educativa en ciencias sociales (Pécaut, 2006, p. 277-409). Ahora bien, de las tensiones al interior del MEN entre profesionales y licenciados por definir los marcos curriculares, salieron victoriosos quienes abogaban por concentrar esfuerzos en los métodos de enseñanza más que en los contenidos propiamente dichos. Esto explica la ambigüedad en las temáticas propuestas, puesto que la nación siguió siendo el eje de las unidades, ya fuese en su relación con el exterior o en su "diversidad interna".

Para el nivel primario se mezclaron asuntos tan diversos como la formación de la nación colombiana, las preocupaciones por la organización regional, los hechos tradicionales de la política decimonónica, el conflicto armado, los derechos humanos y el tráfico de drogas. En secundaria se optó por organizar los temas de acuerdo a la variable temporal, mecanismo que implicaba una visión lineal de la historia desde las civilizaciones antiguas y el mundo prehispánico, pasando por el Medioevo y el Renacimiento, el 
siglo XIX como centuria de los Estados nación hasta la contemporaneidad. Estos periodos históricos se organizarían teniendo en cuenta los tres niveles básicos de la "estructura espacial", a saber: la nación, el continente americano y el mundo (occidental).

Debido al énfasis temático explicitado, el proyecto curricular tendría que hacer hincapié en la promoción y fortalecimiento de habilidades propias de la investigación en las ciencias sociales. Estas destrezas consistían en la formulación de preguntas y problemas, la búsqueda de información, la elaboración y discusión de marcos teóricos, la "operacionalización" de conceptos, la elaboración de hipótesis y la redacción de conclusiones e informes, entre otras. El repertorio de competencias debía tener posibilidades de aplicación para resolver problemas y necesidades concretas, para lo cual sería necesario que el estudiante creara soluciones susceptibles de ser evaluadas de acuerdo al grado y a la fase del desarrollo mental de los alumnos (Abreu, 1988, p. 8-24).

Para cerrar este apartado, se puede citar como ejemplo de la aplicación de los lineamientos generales del proyecto educativo en ciencias sociales el programa curricular para octavo grado de educación secundaria. Realizado por educadores de larga trayectoria, estos le dieron una prelación a la pedagogía activa sobre los contenidos disciplinares, al punto que afirmaron que no se podía pensar en la formación de especialistas. Con base en ello, la contextualización solo debía centrarse en los aspectos relacionados con el "nosotros" nacional. Las "sugerencias metodológicas" se convirtieron en prioridad, con una concepción de las ciencias sociales más cercana a la práctica y a la vivencia de valores democráticos que a la acumulación de conocimientos.

Esto permitió encontrar en los mismos marcos curriculares temas cercanos a una visión leninista de la historia como el estudio del "imperialismo como fase superior del capitalismo", el proceso de formación de las naciones con el fin de crear sentimientos de aprecio hacia la otredad y las bases de la identidad nacional, enfocadas sobre todo hacia el desarrollo socioeconómico y la organización político-administrativa. Aunque la historia política perdió el lugar preponderante de mediados de siglo, los héroes y epopeyas se acompañaron del acercamiento del joven al Estado en su versión republicana y constitucional. En suma, la democracia y el desarrollo fueron los pilares fundamentales de este proyecto educativo al finalizar el siglo XX (Navarro y Ribera, 1990).

\section{A manera de cierre}

Este recorrido por el proyecto educativo del conocimiento social en el sistema educativo colombiano mostró cómo el Estado se ha interesado, por lo menos desde la segunda mitad del 
siglo XX, en la configuración del pasado nacional en el ámbito escolar. Un propósito del Estado nación colombiano por instaurar un sistema de reglas y justificaciones de poder y dominio de unos grupos sociales sobre otros. Como en otras latitudes, este tipo de conocimiento se ha usado para inventar la lealtad y fidelidad a la nación con base en las "glorias" de las generaciones predecesoras, lo que Alarcón Meneses ha llamado para el siglo XIX la "pedagogización del territorio" en el caso del conocimiento geográfico ${ }^{6}$. En el periodo estudiado, se apreció el tránsito lento de una historia encargada de crear sentimientos de obediencia y admiración a los héroes y la patria hacia un relato histórico interesado en mostrar las bondades de la democracia liberal y el sistema de desarrollo capitalista, más allá de los avatares propios del caso colombiano. Este tipo de creación no tiene otro fin que destacar la historia de los héroes y de los grandes prohombres para encontrar lazos de unidad y cohesión nacional. El problema con este enfoque radica en que no establece una línea de demarcación entre el acontecer monumental -propio de toda historia del Estado nación- y la lisonja a los héroes y la ensoñación mística de sus actuaciones. Así, se construye una especie de parodia de la historia con base en la búsqueda de identidades que niegan las tensiones de una sociedad y las formas de dominio y representaciones del poder, esto es, una historia que niega el cambio, la pluralidad $y$, ante todo, que no da cuenta de las transformaciones del saber histórico.

A contrapelo de lo que afirman los funcionarios del MEN cada vez que lanzan una nueva reforma educativa, se evidenció cómo la apuesta por una pedagogía más activa, en la que el estudiante fuese el protagonista, viene por lo menos desde los años sesenta. En ese momento se sentaron las bases para una enseñanza de los "estudios sociales" en los que el activismo pedagógico cobró una relevancia muy fuerte a la que sucumbieron los mismos historiadores profesionales. La expectativa por abandonar la pedagogía tradicional y memorística no reñía con el tipo de contenidos que se contemplaran en los marcos curriculares, máxime cuando se atribuyeron a estos métodos propiedades milagrosas para la formación de ciudadanos democráticos, pacíficos y productivos. Especialmente en el marco de la globalización acelerada de las últimas décadas del siglo XX, en un contexto de neoliberalismo.

6 Alarcón Meneses, L. (2006). La pedagogía territorial y ciudadana en las escuelas republicanas del Caribe colombiano decimonónico. En: Memorias del XIII Congreso Colombiano de Historia. Bucaramanga. 
Al día de hoy todavía el sistema educativo nacional se inscribe en el proyecto de la integración del conocimiento, insistiendo en la defensa de la democracia liberal posterior a la Constitución Política de 1991 y del desarrollo capitalista como metas deseables para la sociedad. No obstante, a diferencia de los días posteriores al 9 de abril de 1948, no se espera mucho de la enseñanza de las ciencias sociales en la escuela, e incluso podría pensarse en que esta área se ha vuelto una pieza de museo en el currículo contemporáneo. A pesar de esta situación, los sellos editoriales privados siguen publicando sus series de manuales, los padres de familia continúan comprando los textos escolares y los estudiantes, a pesar de Wikipedia, todavía los consultan por mandato de sus profesores.

Si el texto escolar aún juega cierto papel en la enseñanza de nuestros días, debiéramos empezar su crítica con el conocimiento de los marcos normativos que de una $u$ otra forma regulan su producción y contenidos. No por casualidad todos los manuales que se encuentran en el mercado aseguran cumplir con lo estipulado en los lineamientos curriculares. Por tal razón, este artículo trató de dar unas puntadas en el estudio sistemático de los proyectos educativos para las ciencias sociales, paso esencial para comprender cómo se ha querido configurar aquello que se debe recordar de nuestro pasado como nación.

\section{Referencias}

Abreu de Armengo, M., et.al. (1988). Marco general de las ciencias sociales-Propuesta de programa curricular sexto grado de educación básica secundaria. Bogotá: MEN.

Acevedo Puello, R. (2011). Memorias, lecciones y representaciones históricas: La celebración del primer centenario de la independencia en las escuelas de la Provincia de Cartagena (1900-1920). Bogotá: Uniandes.

Alarcón Meneses, L. (2006) La pedagogía territorial y ciudadana en las escuelas republicanas del Caribe colombiano decimonónico. En: Memorias del XIII Congreso Colombiano de Historia. Bucaramanga.

Anderson, B. (1993). Comunidades Imaginadas: Reflexiones sobre el origen y la difusión del nacionalismo. México: FCE.

Asociación para la promoción social alternativa Minga y Fundación Progresar. (2008). Memoria: Puerta a la esperanza. Violencia sociopolítica en Tibú y El Tarra Región del Catatumbo 1998-2005. Bogotá: Minga, Progresar, Cisca.

Betancourt, A. (2007). Historia y nación: Tentativas de la escritura de la historia en Colombia. Medellín: La Carreta.

Burgos, C. E., et. al. (1984). Fundamentos generales del currículo. Bogotá: MEN. 
Candau, J. (2002). Antropología de la memoria. Buenos Aires: Nueva Visión.

Comisión Nacional de Reparación y Reconciliación (CNRR) (2010). La Rochela: Memorias de un crimen contra la justicia. Bogotá: Taurus.

Comisión Nacional de Reparación y Reconciliación (CNRR) (2008). Trujillo: Una tragedia que no cesa. Primer informe de memoria histórica de la Comisión Nacional de Reparación y Reconciliación. Bogotá: Planeta.

Dirección general de capacitación y perfeccionamiento docente, currículo y medios educativos-MEN. (1984). Integración Curricular. Bogotá: MEN.

García Samudio, N. La Academia Colombiana de Historia y la enseñanza de la historia patria. Carta dirigida al presidente de la Academia Colombiana de Historia. Noviembre 15 de 1948. En: Ministerio De Educación Nacional. (1949). Enseñanza de la Historia Patria: Normas, estímulos y sanciones. Bogotá: MEN.

Gnecco, C. y Zambrano, M. (2000). Memorias hegemónicas, memorias disidentes: El pasado como política de la historia. Bogotá: Instituto Colombiano de Antropología.

Halbwachs, M. (2004). La memoria colectiva. Zaragoza: Prensas Universitarias de Zaragoza, 2004.

Herrera Bobb, R. y Martínez Guerra, O. (2002) Finalidades y alcances del Decreto 230 del 11 de febrero de 2002: currículo, evaluación y promoción de los educandos, y evaluación institucional. Bogotá: Ministerio de Educación Nacional. Disponible en internet vía: http://www.mineducacion.gov. co/1621/articles-89865_archivo_pdf.pdf. Pp. 17-27.

Herrera, M. (1999). Modernización y Escuela Nueva en Colombia: 1914-1951. Bogotá: UPN/Plaza \& Janés.

Herrera, M., et. al. (2003). La identidad nacional en los textos escolares de ciencias sociales. Colombia 1910-1950. Bogotá: UPN.

Martínez Boom, A. (2004). De la escuela expansiva a la escuela competitiva: Dos modos de modernización en América Latina. Barcelona: Anthropos/CAB, 2004.

Melo, J. y Jaramillo Uribe, J. (1971). Claves para la enseñanza de la Historia. Disponible vía internet: http://www.jorgeorlandomelo.com/calvespara.htm.

Ministerio de Educación Nacional (1949). Resolución No. 1839 de 1948 (9 de octubre). Por la cual se da nombre al Liceo Nacio- 
nal Femenino de Bogotá. En: Ministerio de Educación Nacional. Enseñanza de la Historia Patria: Normas, estímulos y sanciones. Bogotá: MEN.

Ministerio de Educación Nacional. Decreto 088 de 1976 (22 de enero). Por el cual se reestructura el sistema educativo y se reorganiza el Ministerio de Educación Nacional. Disponible en la red vía: http://www.mineducacion.gov. co/1621/articles-102584_archivo_pdf.pdf

Ministerio de Educación Nacional. Decreto 1419 de 1978 (17 de julio). Por el cual se señalan las normas y orientaciones básicas para la administración curricular en los niveles de educación pre-escolar, básica (primaria y secundaria), media vocacional e intermedia profesional. En: Báez Fonseca, Julio Ernesto (Comp.) (1998). Legislación para Educación. 4a Edición. Bogotá: Editorial CASE.

Ministerio de Educación Nacional. Resolución No. 1597 de 1948 (6 de septiembre). Por la cual se disponen los premios para las ganadoras del concurso de historia y se establece la continuidad del concurso. En: Ministerio de Educación Nacional. (1949). Enseñanza de la Historia Patria: Normas, estímulos y sanciones. Bogotá: MEN.

Ministerio de Educación Nacional. Resolución No. 1703 de 1948 (21 de septiembre). Por la cual se adjudican los premios a las ganadoras del concurso de historia. En: Ministerio de Educación Nacional. (1949). Enseñanza de la Historia Patria: Normas, estímulos y sanciones. Bogotá: MEN, 1949.

Ministerio de Educación Nacional. (1963). Programas analíticos de estudios sociales y Filosofía para el primero y segundo ciclos de educación media. Medellín: Bedout.

Navarro Bellido, A. y Ribera Serrano, A. (1990). Ciencias sociales-Marco general. Propuesta programa curricular octavo grado de educación básica. Bogotá: MEN.

Palacios, M. (2003). Entre la legitimidad y la violencia: Colombia, 1875-1994. Bogotá: Norma.

Pécaut, D. (2006). Crónica de cuatro décadas de política colombiana. Bogotá: Norma.

Pérez Garzón, J. et. al. (2000). La gestión de la memoria: La historia de España al servicio del poder. Barcelona: Crítica.

Presidencia de la República. Decreto 2388 de 1948 (15 de julio). Por el cual se intensifica la enseñanza de la historia patria y se dictan otras disposiciones. En: Ministerio de Educación Nacional. (1949). Enseñanza de la Historia Patria: Normas, estímulos y sanciones. Bogotá: MEN.

Presidencia de la República. Decreto No. 3436 de 1948 (6 de octubre). Por el cual se crea la Medalla Cívica "Camilo Torres" 
como homenaje de la República a los educadores más meritorios. En: Ministerio de Educación Nacional. (1949). Enseñanza de la Historia Patria: Normas, estímulos y sanciones. Bogotá: MEN.

Ricoeur, P. (2003). La memoria, la historia, el olvido. Madrid: Trotta.

Rincón, C. et. al. (2010). Entre el olvido y el recuerdo: Íconos, lugares de memoria y cánones de la historia y la literatura en Colombia. Bogotá: Pontificia Universidad Javeriana.

Romero, L. (Coord.) (2004). La Argentina en la escuela: La idea de nación en los textos escolares. Buenos Aires: Siglo XXI.

Sánchez Gómez, G. (2000). Memoria, museo y nación. En: Sánchez Gómez, G. y Willis Obregón, M. (Comps.). Museo, memoria y nación: Misión de los museos nacionales para los ciudadanos del futuro. Bogotá: Ministerio de Cultura/ Museo Nacional de Colombia/PNUD/IEPRI/ICANH.

Sánchez, G. (2006). Guerras, memoria e historia. Medellín: La Carreta.

Tovar Zambrano, B., El pensamiento historiador colombiano sobre la época colonial, En: Anuario Colombiano de Historia Social y de la Cultura (ACHSC) (1982). No. 10, Bogotá, Universidad Nacional de Colombia/Departamento de Historia.

Tovar Zambrano, B. (1994) La historiografía colonial. En: Tovar Zambrano, Bernardo (Comp.). La historia al final del milenio: Ensayos de historiografía colombiana y latinoamericana. Volumen 1, Bogotá, Universidad Nacional de Colombia. p. 21-44.

Velandia, R. (2001). Un siglo de historiografía colombiana: Cien años de la Academia Colombiana de Historia. Bogotá, Academia Colombiana de Historia. 